\title{
Caracterização de serviços de Intervenção e Estimulação Precoce ofertados pelas APAES do estado de São Paulo
}

\author{
Characterization of Early Intervention and Stimulation services offered by \\ APAES of the state of São Paulo
}

Caracterización de los servicios de Intervención Temprana y Estimulación ofrecidos por APAES del estado de São Paulo

\section{Lurian Dionizio Mendonça}

Doutora pela Universidade Estadual Paulista, Bauru, SP, Brasil

E-mail: luriandmendonca@gmail.com ORCID: https://orcid.org/0000-0003-1734-6676

Paula de Marchi Scarpin Hagemann

Doutora pela Universidade Estadual Paulista, Bauru, SP, Brasil

E-mail: pmscarpin@gmail.com ORCID: https://orcid.org/0000-0003-3153-4892

\section{Liene Regina Rossi}

Doutoranda na Universidade Estadual Paulista, Bauru, SP, Brasil

E-mail: lienerossi2016@gmail.com ORCID: https://orcid.org/0000-0003-2365-507X

Olga Maria Piazentin Rolim Rodrigues

Professora adjunta da Universidade Estadual Paulista, São Paulo, SP, Brasil

E-mail: olgarolim@fc.unesp.br ORCID: https://orcid.org/0000-0002-5332-256X

Veronica Aparecida Pereira

Professora associada da Universidade Federal da Grande Dourados, Dourados, MS, Brasil

E-mail: veronicapereira@ufgd.edu.br ORCID: https://orcid.org/0000-0002-9051-7550

Recebido em 19 de setembro de 2019

Aprovado em 18 de dezembro de 2020

Publicado em 20 de janeiro de 2021

\section{RESUMO}

A Intervenção Precoce (IP) ou Estimulação Precoce (EP) pode ser entendida como um conjunto de serviços e apoios, que são necessários à promoção do desenvolvimento pleno de crianças com risco identificado ao nascer ou atraso no desenvolvimento, percebido no decorrer dos primeiros anos, visando melhorar a sua qualidade de vida e de seus familiares. Considerando a importância desse serviço, o presente estudo objetivou caracterizar os serviços de IP/EP ofertados pela Associação de Pais e Amigos dos Excepcionais (APAES) do estado de São Paulo. Foi elaborado, especialmente para este estudo, um protocolo com questões estruturadas, visando obter dados que permitissem caracterizar o serviço ofertado à comunidade. Os resultados apontaram que EP é a terminologia mais utilizada para denominar os serviços, enquanto na literatura o mais frequente é IP, que foi a utilizada nesse estudo. Os profissionais participantes, responsáveis pelo serviço trabalham com IP/EP há muitos anos, mas nenhum relatou pós-graduação neste campo de atuação. Quanto ao atendimento oferecido, predominou o atendimento de crianças com idade 
http://dx.doi.org/10.5902/1984686X61983

superior a quatro anos, com pequeno número de bebês, mostrando que as crianças chegam muito tarde no serviço. No geral, os programas incluem a participação das crianças semanalmente e seus familiares são atendidos esporadicamente. Há presença de equipe multiprofissional, com atendimento interdisciplinar. Ainda que tenha sido relativamente pequeno o número de instituições ouvidas, espera-se que os resultados obtidos com esse estudo impulsionem outros pesquisadores a dar continuidade às pesquisas nessa área tão relevante para o desenvolvimento integral da criança e qualidade de vida para a família.

Palavras-chave: Intervenção Precoce; Estimulação Precoce; infância.

\section{ABSTRACT}

Early Intervention (EI) or Early Stimulation (ES) can be understood as a set of services and supports, which are disseminated to promote the full development of children with identified risk when generating or delayed development, perceived during the first years, Limit improving your quality of life and that of your family members. Regarding the importance of this service, the present study aimed to characterize the El/ES services offered by the Association of Parents and Friends of the Exceptional (APAES) in the state of São Paulo. A protocol with structured questions was elaborated, especially for this study, containing general data that would allow to characterize the service offered to the community. The results pointed out that ES is the most used terminology to name services, while in the literature the most frequent is $\mathrm{El}$, which was used in this study. The participating professionals responsible for the service have been working with El/ES for many years, but none reported postgraduate studies in this field. As for the service offered, predominantly the care of children over the age of four, with the number of children, showing that children arrive very late at the service. In general, the programs include the participation of children on a weekly basis and their families are served sporadically. There is a multidisciplinary team with interdisciplinary care. Although the number of institutions surveyed was relatively small, it is expected that the results obtained with this study will encourage other researchers to continue research in this area, which is so relevant to the integral development of the child and quality of life for the family.

Keywords: Early Intervention; Early Stimulation; childhood.

\section{RESUMEN}

La Intervención Temprana (IT) o Estimulación Temprana (ET) puede entenderse como un conjunto de servicios y apoyos, que se difunden para promover el pleno desarrollo de los niños con riesgo identificado al momento de generar o retrasar el desarrollo, percibido durante los primeros años, Limite la mejora de su calidad de vida y la de los miembros de su familia. En cuanto a la importancia de este servicio, el presente estudio tuvo como objetivo caracterizar los servicios de IT/ET que ofrece la Asociación de Padres y Amigos de los Excepcionales (APAES) en el estado de São Paulo. Se elaboró un protocolo con preguntas estructuradas, especialmente para este estudio, que contiene datos generales que permitirían caracterizar el servicio ofrecido a la comunidad. Los resultados señalaron que ET es la terminología más utilizada para nombrar servicios, mientras que en la literatura la más frecuente es IT, que se utilizó en este estudio. Los profesionales participantes 
responsables del servicio llevan muchos años trabajando con IT/ET, pero ninguno reportó estudios de posgrado en este campo. En cuanto al servicio ofrecido, predomina el cuidado de niños mayores de cuatro años, con el número de niños, lo que demuestra que los niños llegan muy tarde al servicio. En general, los programas incluyen la participación de los niños de manera semanal y sus familias son atendidas de manera esporádica. Existe un equipo multidisciplinario con atención interdisciplinar. Si bien el número de instituciones encuestadas fue relativamente pequeño, se espera que los resultados obtenidos con este estudio incentiven a otros investigadores a continuar investigando en esta área, tan relevante para el desarrollo integral de la niñez y la calidad de vida de la familia.

Palabras clave: Intervención Temprana; Estimulación Temprana; infancia.

\section{Introdução}

Existem evidências na literatura de que o diagnóstico precoce de atrasos no desenvolvimento, seguido de intervenção apropriada, torna possível a prevenção com a consequente diminuição de riscos para o desenvolvimento da criança. A intervenção deve ocorrer, preferencialmente, nos dois primeiros anos de vida pois, em função de sua maior plasticidade neural, a criança responde melhor ao ambiente e às terapias (CARNIEL et al., 2017; HALPERN et al., 2000; RIBEIRO; PEROSA; PADOVANI, 2014).

Há fatores inerentes à criança que podem colocá-la em risco ou vulnerabilidade, como a prematuridade, baixo peso ao nascer, presença de malformações e síndromes (PACHECO, 2013; RODRIGUES; BOLSONI-SILVA, 2011). Outros riscos podem estar associados a fatores psicossociais como: famílias desorganizadas, mães adolescentes e solteiras, abuso de álcool e drogas na gravidez, maus tratos (físico, emocional e abuso sexual), renda familiar insuficiente, baixa escolaridade dos pais, pouca rede de apoio e precariedade dos serviços de saúde (GARCIA et al., 2011; HALPERN et al., 2000). Desse modo, os fatores de risco englobam características intra e extrafamiliares, aumentando a probabilidade das perturbações e atrasos do desenvolvimento (MARTINS, 2004; PACHECO, 2013).

Quanto mais fatores de risco estiverem presentes na vida de uma criança, maior o impacto para seu desenvolvimento. Por isso, destaca-se a importância dos fatores de proteção que podem modificar ou reduzir a repercussão do risco (DUNST, 1995; MARTINS, 2004). Os fatores de proteção são "entendidos como processos dinâmicos nos quais a interação de diferentes fatores produz um fortalecimento do indivíduo" (PACHECO, 2013, p. 39). 
http://dx.doi.org/10.5902/1984686X61983

A Intervenção Precoce (IP) se configura como um fator de proteção. Caracteriza-se como um conjunto de ações necessárias à promoção do desenvolvimento pleno de cada criança, com o objetivo de responder, o mais cedo possível, às suas necessidades. A implementação das ações em IP considera o contexto em que a criança está inserida, reconhecendo as necessidades e potencialidades da família e da comunidade, para analisar e intervir, com a família, sobre os problemas de desenvolvimento (MENDES, 2010).

Para Franco (2007, p. 115), a IP tem um conceito "vasto e dimensional" representado pelo conjunto das intervenções dirigidas às crianças e seus familiares, em contextos de risco ou vulnerabilidade. Os critérios de elegibilidade variam, sendo sugeridos por documentos oficiais ou definidos pela própria instituição prestadora do serviço.

Anteriormente, o modelo de intervenção era denominado Estimulação Precoce (EP), o qual trouxe várias contribuições no âmbito da utilização de padrões desenvolvimentais, descritos em escalas e inventários, que permitiram avaliar comportamentos esperados para a idade da criança, identificar possíveis déficits e promover a estimulação, minimizando ou eliminando possíveis atrasos. Essas competências também foram incorporadas no modelo de IP, porém, o que mudou consideravelmente é o papel do especialista. Enquanto no modelo de EP ele era a figura de referência, por realizar todo processo de avaliação e estimulação, no modelo de IP ele se torna um mediador, tendo a família o papel central. Nessa perspectiva, o especialista tem a função de reconhecer na família suas forças e possibilidades, orientando, ensinando e potencializando ações para que ela realize, em contexto natural, as intervenções necessárias ao desenvolvimento da criança (SIMEONSSON; PEREIRA; SCARBOROUGH, 2003).

Embora a diferença dos modelos de EP e IP sejam fundamentais, é possível que o longo tempo de utilização do termo estimulação e, até mesmo, o poder centrado na figura do especialista como detentor da ação, apresenta-se como um dificultador para utilização da nova terminologia e, consequentemente, de seu modelo, levando a incompreensões que por vezes, indicarão os termos como sinônimos. Segundo Franco (2007), a denominação inicial foi EP centrada na criança e, especificamente, no desenvolvimento motor e sensorial. Para o autor, o conceito de IP é mais amplo, centrado na família, compreendendo todo o conjunto de intervenções que precisam ser promovidas até os seis anos, com o objetivo de atender "às necessidades transitórias ou permanentes que apresentam" (p. 115).

Apesar da dificuldade de se implementar intervenções precoces em países em desenvolvimento, como é o caso do Brasil, isto não se torna um impedimento para tal. É 
http://dx.doi.org/10.5902/1984686X61983

possível realizar programas de baixo custo que podem cumprir essa função, tais como: visita domiciliar com o objetivo de orientar os pais na estimulação dos filhos, formação de cuidadores e professores nas escolas e creches, e divulgação de orientações sobre desenvolvimento infantil nos meios de comunicação (HALPERN et al., 2000).

Contudo, nos documentos oficiais brasileiros, o termo que se encontra descrito é EP, preconizando o atendimento de crianças até três anos de idade, com o objetivo de oferecer o mais cedo possível intervenções voltadas ao contexto das famílias, de modo a atender às suas necessidades, transitórias ou permanentes (BRASIL, 2016). Embora o documento considere o contexto das famílias, ainda permanece a ideia de que serão atendidas por um especialista que deve realizar a intervenção, sem destaque para o potencial da família e comunidade para a promoção do desenvolvimento. Além disso, limita a intervenção até os três anos de idade.

A adoção da EP determina não só o nome, mas como os serviços são oferecidos à população, conforme definido pelo Ministério da Saúde (Brasil, 2016):

[...] um programa de acompanhamento e intervenção clínico-terapêutica multiprofissional com bebês de alto risco e com crianças pequenas acometidas por patologias orgânicas, buscando o melhor desenvolvimento possível, por meio da mitigação de sequelas do desenvolvimento neuropsicomotor, bem como de efeitos na aquisição da linguagem, na socialização e na estruturação subjetiva, podendo contribuir, inclusive, na estruturação do vínculo mãe/bebê e na compreensão e no acolhimento familiar dessas crianças (p. 7).

A proposta de oferta dos serviços de EP é importante, como já destacado. Entretanto, ao limitar sua atuação até os três anos e ao centrar sua atenção sobre a criança, restringe seu alcance não só em relação ao número de pessoas atendidas como à continuidade da intervenção. Tegethof (2007) relata que a IP, como um conjunto de serviços e apoios, pode ser promovida em diferentes contextos, tendo como base uma relação de parceria com a família. Sua oferta, além de possibilitar a promoção do desenvolvimento das crianças, em idades precoces, com deficiências, risco ou atraso de desenvolvimento, visa melhorar a sua qualidade de vida, assim como a de suas famílias. Porém, conforme destacado por Schmidt et al. (2015), ao buscarem identificar programas de IP voltados às crianças com autismo, identificaram poucos estudos na literatura nacional. Os autores destacaram a importância da intervenção com a família, em contexto natural, implementando mudanças de rotinas e investindo na aquisição de habilidades importantes para identificar e atender as necessidades da criança, melhorando a qualidade de vida da família. 
http://dx.doi.org/10.5902/1984686X61983

De acordo com Pimentel (2004), a IP inclui todo o tipo de atividades e procedimentos destinados a promover o desenvolvimento e aprendizagem da criança, assim como o conjunto de oportunidades para que as famílias possam promover esse mesmo desenvolvimento e aprendizagem. Portanto, todas as ações e serviços que forem ofertados a essas crianças, deverão incluir seus familiares. Para que isso ocorra, a autora destaca que é preciso construir uma relação de confiança e respeito entre os familiares e os profissionais da equipe de intervenção, pois ainda hoje, os pais não estão acostumados a fazer parte dos serviços e atendimentos pelos quais seus filhos passam. Bolsanello (2003) também mostrou, em seu estudo, a pouca participação dos pais no processo.

A opção pelo modelo de EP pode resultar de experiências que centram na figura do especialista o controle sobre os processos de tomada decisão, tendo como base sua competência profissional, em uma relação assimétrica com a família, como se ela não fosse capaz de identificar o que a criança precisa e atender suas necessidades. Nessa condição, a família se restringe a expectadora ou cumpridora de regras (DALMAU et al., 2017). No modelo de IP a participação da família é fundamental, tendo - mediador a função de empoderar as famílias sobre práticas de cuidado, acompanhamento e orientação de seus filhos para independência e autonomia, considerando as possibilidades e necessidades da família e da criança (DALMAU et al., 2017; FRANCO, 2007).

Segundo Almeida et al. (2011), os serviços de IP devem ser criados em resposta às necessidades específicas das crianças e de suas famílias e, sempre que possível, devem ser integrados nas atividades diárias dessas crianças. Para tanto, devem ocorrer em seu ambiente natural, no qual elas passam a maior parte de seu tempo. As autoras destacaram, ainda, que práticas de intervenção baseadas na rotina diária dessas crianças como banho, alimentação e hora de dormir, são oportunidades essenciais de ensino e aprendizagem. García, Madrid e Galante (2017) ao descreverem programas de IP com oito famílias, destacaram a relevância da intervenção para aquisição e aprimoramento de competências em relação à comunicação entre pais e filhos, os reflexos em relação a diminuição de estresse e o aumento de competências das famílias para resolução de problemas de forma autônoma. Nesta direção, Correa, Minetto e Crepaldi (2017), em estudo de revisão bibliográfica, apontaram a contribuição da IP no direcionamento de ações voltadas à família, à comunidade e à valorização do trabalho em equipe, destacando o potencial das famílias como promotoras de desenvolvimento. 
http://dx.doi.org/10.5902/1984686X61983

No Brasil, uma das referências quando refere-se a reabilitação de indivíduos com alguma deficiência ou atraso no desenvolvimento, é a Associação de Pais e Amigos dos Excepcionais (APAE). Ela está presente em todos os estados e em inúmeros municípios. No estado de São Paulo, as APAES são representadas pela Federação das APAES do Estado de São Paulo (FEAPAES-SP). A FEAPAES-SP atua há 25 anos no assessoramento das 305 APAES existentes em todo o estado e em defesa das pessoas com deficiência intelectual e múltipla (FEAPAES-SP, 2019). Historicamente, é considerado um serviço de referência, de caráter filantrópico, mantido prioritariamente com recursos públicos (RAFANTE; SILVA; CAIADO, 2019), assumindo um serviço que seria dever do estado. Todavia, por ser a principal oferta de serviço para reabilitação de pessoas com deficiência, os usuários demonstram confiança na atuação de seus profissionais (GARCIA, 2006). É possível observar publicações em relação a EP (BOBREK; GIL, 2016; GOLLO; GRAVE, 2015) e em IP (FERREIRA et al., 2019; IZIDORO et al., 2019; ORTIZ; RIBEIRO, 2013), realizadas com os profissionais que atuam nas APAES, bem como ao público atendido nessas instituições, sejam crianças prematuras, com baixo peso ao nascer ou, ainda, com outras necessidades identificadas, que se beneficiariam do serviço.

Gollo e Grave (2015), em estudo que antecedeu as diretrizes, caracterizaram a EP como serviço destinado às crianças com deficiência ou risco até quatro anos (e não três como a atual diretriz). Identificaram a presença de EP nas cinco APAES existentes no Vale do Taquari (SP), com a atuação de profissionais das áreas de Fisioterapia, Fonoaudiologia, Terapia Ocupacional e Pedagogia (ocorrência simultânea de até três áreas, dado contraditório para instituições que indicaram apenas um profissional atuando em EP). As autoras destacaram a importância das ações em EP e as contribuições para a criança com deficiência, indicando a relevância do profissional especializado para orientações aos pais.

Bobrek e Gil (2016) investigaram a percepção de 13 profissionais, de três diferentes instituições, acerca das relações estabelecidas em sua prática em EP e a formação durante a graduação. As autoras identificaram que os entrevistados reconhecem a EP como oferecimento de oportunidades para o desenvolvimento da criança e/ou suporte à família ou prevenção de risco para o desenvolvimento. Contudo, os entrevistados relataram também dificuldades acerca da falta de envolvimento familiar e falta de infraestrutura para desenvolver atividades específicas com a criança.

No estudo qualitativo realizado por Ortiz e Ribeiro (2013) com as APAES, buscou-se conhecer a percepção das mães de bebês e crianças com síndrome de Down em relação 
http://dx.doi.org/10.5902/1984686X61983

ao processo de reabilitação dos filhos que participavam de um programa de IP na APAE de Goiânia. Como resultado, identificaram que as mães entrevistadas relataram estarem satisfeitas com o desenvolvimento que os filhos alcançaram a partir do programa de IP. Além disso, as autoras destacaram a importância da IP para o desenvolvimento neuropsicomotor e social de um bebê com síndrome de Down, repercutindo diretamente na estrutura familiar.

Izidoro et al. (2019) buscaram caracterizar a ocorrência de IP nas APAES, no estado do Mato Grosso do Sul. Com a participação de $20 \%$ das instituições existentes, as autoras relataram que, embora os profissionais que responderam à pesquisa tenham relatado a oferta de serviços de IP, a descrição das ações encontra-se fundamentada em EP, com baixa participação familiar e serviços centrados na criança. As autoras destacaram a importância de formação continuada, de modo a evidenciar a necessidade de atuar junto às famílias, com intervenções inseridas em seu contexto e desenvolvimento de ações que possam favorecer a participação e envolvimento.

Situação semelhante foi indicada por Ferreira et al. (2019), ao investigar sobre a participação da família nos serviços de IP das APAES do estado de São Paulo. As autoras verificaram que, apesar dos profissionais atuantes nas APAES valorizarem essa participação, na maioria dos casos o atendimento às famílias só é realizado quando é identificado alguma necessidade específica da criança. Os dados corroboram os achados de Izidoro et al. (2019) indicando que mesmo quando os serviços são nomeados como IP, não são centrados na família e sim na criança, estando ainda arraigados no modelo de EP.

Em seu estudo, Nascimento et al. (2018) buscaram caracterizar as instituições que prestam serviços de IP, na cidade de Curitiba (PR), e os profissionais que atuam nesse serviço. Os resultados mostraram que as instituições do terceiro setor são as mais atuantes nessa área, atendendo crianças na faixa etária de zero a três anos de idade e, os profissionais mais atuantes nas equipes de IP são professores, pedagogos e psicólogos. Considerando os apontamentos de Perez-Lopes (2008), a atuação profissional em IP requer um campo mais amplo de atuação profissional, contando com as áreas de Medicina, Psicologia, Terapia Ocupacional, Fisioterapia, Fonoaudiologia e Pedagogia podendo, ainda, ser complementado com outras áreas, destacando as competências acerca do conhecimento e em relação: a) ao desenvolvimento infantil (em especial de zero a seis anos); b) às patologias da infância; c) aos efeitos do ambiente sobre o desenvolvimento infantil; d) aos instrumentos de avaliação de desenvolvimento (gerais e específicos) e, e) 
http://dx.doi.org/10.5902/1984686X61983

ao desenvolvimento de habilidades para o trabalho transdisciplinar. Os profissionais que atuam em equipes de IP deverão ser capazes de, sistematicamente, planejar, reavaliar e replanejar nos diferentes contextos de desenvolvimento.

Nesse estudo, optou-se por manter a terminologia IP, por considerá-la mais abrangente, focalizando, não só as necessidades da criança, como também de sua família. De modo mais amplo, os processos de desenvolvimento são compreendidos na perspectiva do modelo transacional de Sameroff e Fiese (1990). Nesse modelo, as interações familiares são percebidas de forma bidirecional, compreendendo que as forças e fragilidades inerentes à criança que chega a uma família interferem diretamente no funcionamento da mesma, de modo recíproco. Por isso, ao atuar em IP, não é só a criança que se apresenta para a proposição de avaliações e intervenções, mas toda a família, com sua história, medos, anseios, habilidades e fragilidades.

Devido à importância da IP para o desenvolvimento de crianças de risco e suas famílias, justifica-se a necessidade de se compreender como esses serviços são oferecidos enquanto rede de apoio da Educação Especial. Apesar dos bons resultados obtidos com a IP descritos nos estudos encontrados, observa-se uma escassez dos mesmos, principalmente no que se refere à realidade brasileira (SCHMIDT et al., 2015). Descrever como esse serviço acontece nas APAES do estado de São Paulo, pode facilitar o aprimoramento do serviço, o encaminhamento de mais crianças e o oferecimento de cursos de formação continuada, se necessário, entre outras ações. Desse modo, o presente estudo teve como objetivo caracterizar os serviços de IP ofertados pelas APAES do estado de São Paulo.

\section{Método}

Trata-se de um estudo com delineamento transversal e descritivo. O mesmo foi aprovado pelo Comitê de Ética da Faculdade de Ciências, da UNESP de Bauru, via Plataforma Brasil (CAAE: 68608317.6.0000.5398). Os participantes assinaram o Termo de Consentimento Livre e Esclarecido, conforme estabelece a Resolução 466/2012 (BRASIL, 2012) do Conselho Nacional de Saúde. Para os respondentes foi garantido sigilo das informações suas e da instituição explicitando que os dados seriam analisados em bloco, impossibilitando a identificação de ambos. 


\section{Participantes}

Foram convidadas a participar dessa pesquisa $184(60,32 \%)$ das 305 APAES do estado de São Paulo que atenderam ao critério estabelecido nesse estudo, ou seja, aquelas que após contato inicial, via telefone, indicaram a existência de serviço de IP/EP na instituição.

\section{Instrumentos}

Foi elaborado, especialmente para esse estudo, um questionário com questões estruturadas visando obter dados que permitissem caracterizar os serviços de IP oferecidos pelas APAES no que se refere ao tempo de existência do serviço, às categorias profissionais incluídas, critérios de elegibilidade, realização de reuniões, dificuldades, origem dos encaminhamentos, dentre outros. No questionário foi acrescida a terminologia EP por fazer parte dos documentos legais (BRASIL, 2016), pelo fato da literatura indicar a ocorrência dos dois modelos (BOBREK; GIL, 2016; FERREIRA et al., 2019; GOLLO; GRAVE, 2015; IZIDORO et al., 2019; ORTIZ; RIBEIRO, 2013) e, por perceber, via contato telefônico inicial, que o termo EP era o mais conhecido pelas instituições.

\section{Procedimentos}

A partir do site da FEAPAES-SP (http://www.feapaesp.org.br) obteve-se a lista das APAES do estado de São Paulo com respectivos e-mails e telefones. Inicialmente, as APAES foram indagadas, via telefone, sobre a existência de um serviço de IP/EP. As que responderam afirmativamente a esta questão, foram esclarecidas quanto aos objetivos do estudo e foram convidadas a colaborar com o mesmo. Na sequência, receberam por e-mail um questionário com questões relacionadas à caracterização do serviço oferecido. Os questionários para a coleta de dados tiveram um prazo máximo de 15 dias para devolução.

\section{Análise dos dados}

Para a análise dos dados, as respostas obtidas com os questionários foram categorizadas e inseridas em um banco de dados. Foi empreendida análise descritiva, com checagem de consistência dos dados. Os dados estão apresentados em frequências, em média ou mediana e desvio padrão, quando apropriado. 


\section{Resultados}

Das 305 APAES contatadas, 184 responderam afirmativamente quando questionadas sobre a existência de um serviço de IP/EP. Entre a respondentes, apenas $22(11,95 \%)$ devolveram o questionário dentro do prazo estipulado.

Dos questionários recebidos nem todas as questões foram respondidas por todos os participantes. Em cada um dos dados analisados estão a quantidade de respostas referentes aos itens. A totalidade dos respondentes desse estudo é constituída por mulheres, com média de idade de 42,5 anos ( $D P=11,2$ ), com formação predominante em Psicologia (28,6\%). Nenhuma das participantes relatou ter realizado algum curso de pós-graduação em IP/EP. Com relação ao tempo de trabalho na APAE, o tempo médio de meses trabalhados foi de 163 meses, sendo que $36,8 \%$ têm como função atividades relacionadas à sua área de atuação dedicando-se, principalmente, a orientação e atendimento de crianças e seus pais. Com relação ao tempo de atuação com IP/EP, a mediana de tempo de trabalho foi de 60 meses e, a principal função também foi de atividades relacionadas à sua área de atuação $(50 \%, n=10)$, seguida por coordenação de serviços de saúde/gestão $(45 \%, n=9)$. Esses dados podem ser observados na Tabela 1.

No que diz respeito às características dos serviços de IP/EP oferecidos pelas APAES participantes do presente estudo, $68,2 \%(n=15)$ denominaram o serviço ofertado de Estimulação Precoce, seguido por 13,6\% como Educação Precoce, 9,1\% ( $n=2)$ designando como Intervenção Precoce, 4,5\% como Interação Essencial $(n=1)$ e Estimulação Essencial $(n=1)$. Já com relação ao tempo de existência do serviço, 52,4\% (n $=11$ ) eram serviços com mais de 20 anos de existência, com tempo total oscilando entre 22 e 33 anos. Os demais serviços eram mais recentes, visto que $28,6 \%(n=6)$ relataram atuar há mais de cinco anos e, 9,5\% há menos de cinco anos. 
Tabela 1 - Dados sociodemográficos das respondentes

\begin{tabular}{c|c|c}
\hline FORMAÇÃo ACADÊMICA & $\%$ & $\mathbf{n}=\mathbf{2 1}$ \\
\hline Psicologia & 28,6 & 6 \\
\hline Terapia Ocupacional & 19 & 4 \\
\hline Pedagogia & 19 & 4 \\
\hline Fisioterapia & 14,3 & 3 \\
\hline Serviço Social & 9,5 & 2 \\
\hline Fonoaudiologia & 4,8 & 1 \\
\hline Enfermagem & 4,8 & 1 \\
\hline FUNÇÃo NA APAE & $\%$ & $\mathbf{n}=\mathbf{1 9}$ \\
\hline Atendimento a pais e alunos, orientação na área de atuação \\
profissional & 26,3 & 5 \\
\hline Educação & 36,8 & 7 \\
\hline Gestão/coordenação e área de atuação profissional & 26,3 & 5 \\
\hline FUNÇÃo NA IP & $\%$ & $\mathbf{n}=\mathbf{2 0}$ \\
\hline Gestão/Coordenação de serviço & 45 & 9 \\
\hline Atendimento a pais e alunos, orientação na área de atuação & 50 & 10 \\
\hline profissional & 5 & 1 \\
\hline Educação & & 2 \\
\hline
\end{tabular}

Fonte: Elaborado pelas autoras (2019).

A Tabela 2 apresenta dados relacionados a faixa etária atendida e o número de crianças atendidas em cada uma delas. Com relação à faixa etária das crianças, 33,3\% (n = 7) afirmaram atender crianças entre zero e quatro anos de idade. Todavia, relataram maior frequência de atendimento na faixa etária acima de quatro anos de idade $(43,4 \%)$.

No que diz respeito ao processo de chegada aos serviços de IP/EP, 81,8\% ( $n=18)$ das APAES, recebem as crianças via encaminhamento de hospitais, serviços de saúde do município, central de vagas e demanda dos pais. Para as demais, os encaminhamentos são provenientes apenas de hospitais $(9,1 \%, n=2)$ e serviços de saúde do município $(9,1 \%$, $n=2)$. Ao receber esses encaminhamentos, $95,5 \%(n=21)$ das APAES participantes mencionaram ter um serviço de triagem. 
Tabela 2 - Faixa etária das crianças atendidas

\begin{tabular}{l|c|c}
\hline \multicolumn{1}{c|}{$\begin{array}{c}\text { Faixa etária das crianças atendidas, segundo as } \\
\text { respondentes }\end{array}$} & $\%$ & $\mathbf{n}=\mathbf{2 1}$ \\
\hline 0 a 3 anos & 28,6 & 6 \\
\hline 3 a 4 anos & 33,3 & 7 \\
\hline 4 a 5 anos & 14,3 & 3 \\
\hline 5 a 6 anos & 23,8 & 5 \\
\hline Idade das crianças atendidas atualmente, em números & $\%$ & $\mathbf{n = 2 0}$ \\
\hline Bebês de 0 a 12 meses & 7,5 & 94 \\
\hline Bebês de 13 a 24 meses & 16,7 & 210 \\
\hline Crianças de 25 a 36 meses & 18,8 & 236 \\
\hline Crianças de 37 a 48 meses & 14,6 & 184 \\
\hline Crianças de 49 meses ou mais & 42,4 & 534 \\
\hline
\end{tabular}

Fonte: Elaborado pelas autoras (2019).

Com relação à triagem, $38,1 \%(n=8)$ referiu ser realizada por uma equipe multiprofissional constituída, geralmente, por psicólogo, assistente social, fisioterapeuta, terapeuta ocupacional, fonoaudiólogo e pedagogo. Os dados referentes ao processo de triagem encontram-se na Tabela 3.

Tabela 3 - Processo de triagem das APAES para o serviço de IP

\begin{tabular}{l|c|c}
\hline \multicolumn{1}{c|}{ Triagem } & $\%$ & $\mathbf{n = 2 1}$ \\
\hline $\begin{array}{l}\text { Anamnese inicial, seguido de avaliação da equipe } \\
\text { multiprofissional }\end{array}$ & 33,3 & 7 \\
\hline Avaliação por equipe multiprofissional & 38,1 & 8 \\
\hline $\begin{array}{l}\text { Triagem pelo Serviço Social } \\
\text { Avaliação Médica, seguido por avaliação da equipe } \\
\text { multiprofissional }\end{array}$ & 23,8 & 5 \\
\hline
\end{tabular}

Fonte: Elaborado pelas autoras (2019).

Observa-se que em 38,1\% dos serviços, a triagem é feita pela equipe de profissionais. No restante, inicialmente passa por um profissional (médico ou assistente social) que, provavelmente, define quem será encaminhado para avaliação da equipe, isto é, os elegíveis para o serviço. 
http://dx.doi.org/10.5902/1984686X61983

Os resultados referentes às condições do bebê que são critérios de elegibilidade para participar do programa de IP/EP encontram-se na Tabela 4. Os mais frequentes foram prematuros, sindrômicos, doença grave, malformação $(52,4 \%)$ e, para $23,8 \%$ foram incluídos os bebês prematuros de alto risco, bebê com baixo peso ao nascer e com risco social.

Tabela 4 - Critérios de elegibilidade para o bebê participar do programa de IP

\begin{tabular}{l|c|c}
\hline \multicolumn{1}{c|}{ Critérios de elegibilidade } & $\%$ & $\mathbf{n = 2 1}$ \\
\hline $\begin{array}{l}\text { Prematuros e prematuros de alto risco, bebê com muito baixo } \\
\text { peso, sindrômicos, bebês com risco social, doenças graves, } \\
\text { malformação }\end{array}$ & 23,8 & 5 \\
\hline Prematuros, baixo peso, risco social & 4,8 & 1 \\
\hline Sindrômicos, doença grave, malformação & 9,5 & 2 \\
\hline Prematuros, sindrômicos, doença grave, malformação & 52,4 & 11 \\
\hline Outros & 9,5 & 2 \\
\hline
\end{tabular}

Fonte: Elaborado pelas autoras (2019).

No que se refere aos programas de IP/EP ofertados, a participação prioritária nos programas é voltada para crianças e cuidadores (pais e/ou responsável) em 86,4\% ( $n$ = 19). Ao serem indagados sobre como acontece a participação dos pais e/ou responsáveis, $68,2 \%(n=15)$ responderam que acontece desde a triagem e, 31,8\% $(n=7)$ que acontece nos serviços disponibilizados e continua em casa. Quanto à periodicidade dos atendimentos oferecidos aos bebês, $61,9 \%(n=13)$ das respostas válidas referiram que esse era semanal e envolvia todas as áreas. Já com relação à periodicidade dos atendimentos oferecidos aos pais, $45,5 \%(n=10)$ responderam que também era semanal e envolvia todas as áreas (pediatria, psicologia, fisioterapia, fonoaudiologia, terapia ocupacional, pedagogia). Os resultados referentes à periodicidade dos atendimentos oferecidos aos bebês e aos pais estão apresentados na Tabela 5.

No que se refere ao fato da IP/EP ser ofertada em outros locais além da APAE, 54,5\% ( $n=12)$ disseram que sim. Com relação a esses lugares, dentre as respostas válidas, $16,7 \%(n=2)$ disseram que acontece em visitas periódicas sistemáticas à residência da criança, 41,7\% ( $n=5)$ em visitas periódicas sistemáticas à creche/escola que frequenta e, $41,7 \%(n=5)$ em visitas periódicas sistemáticas à residência da criança e à escola/creche. 
http://dx.doi.org/10.5902/1984686X61983

Tabela 5 - Periodicidade dos atendimentos oferecidos às crianças e às famílias

\begin{tabular}{l|c|c}
\hline \multicolumn{1}{c|}{ Periodicidade dos atendimentos oferecidos aos bebês } & $\mathbf{\%}$ & $\mathbf{n = 2 1}$ \\
\hline Mais de uma vez por semana, em diferentes áreas & 33,3 & 7 \\
\hline Semanal, envolvendo todas as áreas & 61,9 & 13 \\
\hline De acordo com a necessidade identificada na triagem & 4,8 & 1 \\
\hline \multicolumn{1}{c|}{ Periodicidade dos atendimentos oferecidos aos pais } & $\%$ & $\mathbf{n = 2 2}$ \\
\hline Mais de uma vez por semana & 18,2 & 4 \\
\hline Semanal, envolvendo todas as áreas & 45,5 & 10 \\
\hline Quinzenal, envolvendo todas as áreas & 4,5 & 1 \\
\hline Mensal, envolvendo todas as áreas & 9,1 & 2 \\
\hline Não são oferecidos atendimentos aos pais & 9,1 & 2 \\
\hline Atendimentos quinzenais com palestras aos pais & 4,5 & 1 \\
\hline Atendimentos mensais com palestras aos pais & 4,5 & 1 \\
\hline De acordo com a necessidade identificada na triagem & 4,5 & 1 \\
\hline
\end{tabular}

Fonte: Elaborado pelas autoras (2019).

\section{Discussão}

No presente estudo buscou-se caracterizar os serviços de IP/EP ofertados pelas APAES do estado de São Paulo, por meio de respostas obtidas em um questionário elaborado para este fim. Das 184 APAES que afirmaram, inicialmente, oferecer esse tipo de serviço, apenas 22 responderam ao questionário dentro do prazo estabelecido. Logo, os dados, apesar de trazerem informações importantes e pertinentes, não são passíveis de generalização.

No que se refere ao perfil das respondentes do presente estudo, observa-se que diferentes profissionais são responsáveis pela coordenação do serviço de IP/EP, mostrando que, independente da especialidade, são igualmente envolvidos, não observando predominância de uma das áreas na realização da intervenção. Contudo, não foi possível identificar o quanto essas diferentes áreas atuam entre si, o que é um pressuposto importante para o trabalho transdisciplinar, conforme apontado por Franco (2007). O tempo médio de atuação dos profissionais (13 anos) pode ser um indicativo de maturidade na profissão. Atuar junto a profissionais experientes pode ser positivo em relação à manutenção de práticas que se mostram efetivas, mas pode também ser um 
http://dx.doi.org/10.5902/1984686X61983

complicador quando esses demonstram pouca abertura para aprendizagem e necessidades de mudança. Esse pode ser um dos fatores associados aos achados de Ferreira et al. (2019) e Izidoro et al. (2019) quando os participantes nomeiam suas práticas como IP e continuam realizando EP. Essa possível acomodação em torno de práticas já estabelecidas pode ser reafirmada quando nenhum deles relatou formação específica de pós-graduação em IP ou áreas afins.

Ainda que os profissionais não tenham sido convidados a avaliar sua própria formação, destaca-se os resultados obtidos por Bolsanello (2003), em que os próprios profissionais quando avaliaram o seu desempenho profissional o caracterizaram como deficitário, atribuindo, principalmente, à sua formação, uma vez que, a maioria dos profissionais não tinha curso específico na área e os que tinham, disseram que o curso havia sido centralizado somente no fornecimento de estimulação à criança. Ainda que passados 15 anos após esse estudo, a realidade encontrada não foi diferente, pois nenhum participante disse ter curso de pós-graduação em IP/EP, o que reforça a hipótese anteriormente levantada.

Quanto a denominação do serviço observou-se o uso mais frequente da terminologia EP. Uma hipótese levantada é que, mais do que associada à uma filosofia de atendimento mais abrangente, pode estar associada a nomenclatura utilizada nos documentos oficiais e práticas reafirmadas ao longo dos anos. Almeida et al. (2011), destacaram que a terminologia deveria estar atrelada ao que o serviço oferece e, que a IP deveria ser implementada com o objetivo de gerar efeitos benéficos tanto para as crianças como para seus familiares. Envolver a família nesse processo é algo que pode inicialmente impor resistências, tanto por parte dos profissionais como da própria família. Contudo, na medida em que os avanços são observados, tais com a diminuição do estresse, avanço na comunicação, independência e autonomia, aumenta-se o envolvimento e compromisso de todos em busca de qualidade da vida para a criança e sua família (CORREA; MINETTO; CREPALDI, 2017; GARCÍA; MADRID; GALANTE, 2017).

A respeito da faixa etária das crianças atendidas, verificou-se que o percentual indicado pela diretriz, até três anos, é observado para a minoria (28,6\%). Observou-se predominância de atendimentos a crianças com 49 meses de vida ou mais, o que permite levantar duas possíveis hipóteses: primeiro, que as crianças chegam ao serviço tardiamente, fora do período que poderiam ser mais beneficiadas pelo serviço de IP (CARNIEL et al., 2017; PADOVANI et al., 2014). A segunda hipótese traz um indicativo de 
http://dx.doi.org/10.5902/1984686X61983

sensibilidade da instituição para a necessidade de continuidade dos serviços, o que se alinha às orientações internacionais (FRANCO, 2007; PEREZ-LOPES, 2008). Esse dado precisa ser melhor investigado, seja no sentido de efetivar diagnóstico precoce, visto que quanto mais cedo se iniciarem os programas de IP, mais eficazes serão as intervenções (BAIRRÃO; ALMEIDA, 2003), ou seja, no sentido de provocar políticas públicas que ampliem a obrigatoriedade de serviços de IP até os seis anos de idade. Os resultados desse estudo mostraram que apenas $7,5 \%$ das APAES respondentes tinham, em atendimento, bebês menores de um ano de idade. Contudo, esse fato pode ser decorrente da dificuldade e demora no encaminhamento para esse serviço, uma vez que, para intervir precocemente é preciso identificar e orientar profissionais da saúde para realizar o encaminhamento o mais cedo possível, e incentivando os pais para a adesão ao serviço (FRANCO, 2007; SOARES et al., 2012).

Quanto ao critério de elegibilidade, ainda não é previsto, para a maioria dos serviços, a inclusão de bebês/crianças com risco social, o que caracterizaria um serviço de IP centrado na família. Mais de $50 \%$ consideraram como critério apenas crianças prematuras, com alguma síndrome, doença grave ou malformação, aparentemente serviços focados mais nas condições da criança. Soares et al. (2012) definem que uma criança em risco de desenvolvimento não é apenas aquela que apresenta traços de anormalidade, mas todas aquelas que possam estar expostas a fatores biológicos ou ambientais sugestivos, o que significaria um trabalho intenso e amplo com a família, lócus principal de desenvolvimento da criança. Tais crianças deveriam ser incluídas nesses atendimentos, mostrando que o problema pode estar antes da intervenção, já na identificação, encaminhamento e triagem, fato esse que deve ser mais apurado em novos estudos com dados de outras instituições que oferecem esse tipo de serviço.

A identificação da clientela para os programas de IP é feita, prioritariamente, por profissionais da saúde de diferentes serviços, como maternidades e núcleos de saúde. É preciso sensibilizá-los para o encaminhamento precoce, esclarecendo a importância desse tipo de atendimento. Quando na instituição, acredita-se que um dos fatores que pode auxiliar na elegibilidade da clientela é a existência de uma equipe multiprofissional, na qual, cada profissional tem o seu saber, a sua especificidade, mas que se complementam (BORTAGARAl et al., 2015; FRANCO, 2007). Bolsanello (2003), também apontou que uma das falhas na formação desse profissional é a habilidade em realizar trabalhos em equipe e, consequentemente, na troca de experiências entre os pares, observando que, cada 
http://dx.doi.org/10.5902/1984686X61983

profissional se preocupa somente em realizar as atividades que são específicas da sua área, sem contextualização caracterizando, assim, uma equipe multiprofissional e não inter ou o mais adequado, transdisciplinar. Perez-Lopes (2008) enfatizou a necessidade de conhecimentos específicos em desenvolvimento infantil e em outros aspectos diretamente relacionados à IP. No presente estudo, a formação predominante dos profissionais foi em Psicologia e os respondentes atribuíram como principal área de atuação na APAE a orientação e o atendimento as crianças e seus familiares, dentro do seu campo específico de atuação. Essa é uma característica de atendimentos interdisciplinares quando os profissionais estão todos no mesmo contexto, mas os atendimentos são realizados separadamente. Franco (2007) indicou a relevância do modelo de atendimento transdisciplinar, no qual todos os profissionais são corresponsáveis pela implementação do programa, independente de quem irá executá-lo. Os resultados são mais visíveis pela família, que também é parte integrante da equipe.

Um ponto de destaque observado é que mais de $80 \%$ dos programas de IP analisados incluíam a participação das crianças e seus familiares. Entretanto, o que deve ser analisado em futuros estudos é a forma como os familiares são incluídos nos atendimentos. Assim como indicado por Almeida et al. (2011), acerca da falta de um envolvimento ativo das famílias nas escolhas e tomadas de decisão durante o processo de avaliação e intervenção, os dados do presente estudo sugerem baixa participação da família no processo de IP. Uma abordagem centrada na família considera como alvo de intervenção não só a criança, mas toda a família, visto que o bem-estar de um membro poderá influenciar todos os demais (SOARES et al., 2012).

A IP centrada na família e seu contexto, favorece a atuação preventiva, de modo a viabilizar melhor qualidade de vida para toda a família (HANSEL; BOLSANELLO, 2012; SAMEROFF; FIESE, 1990). Soares et al. (2012) apontaram que cada serviço de IP deve ser individualizado e estruturado com base na criança e sua família, considerando sua individualidade, forças, necessidades e potencialidades. Ao analisar a periodicidade dos atendimentos, com predomínio de ações uma vez por semana, percebe-se que essa frequência pode estar mais relacionada à realidade institucional do que às necessidades das famílias, que podem ter planejamentos muito distintos, requerendo para tanto a presença de um mediador, representante da equipe, em contexto natural.

Contudo, vale ressaltar que o presente estudo visou caracterizar como funcionam os serviços de IP/EP ofertados pelas APAES do estado de São Paulo e não a avaliação desse 
http://dx.doi.org/10.5902/1984686X61983

serviço. No entanto, a avaliação é de fundamental importância. Bairrão e Almeida (2003), apontaram que a avaliação de programas de IP não é uma tarefa fácil, devido a sua complexidade, por ter um caráter individual e abrangente, pois engloba, a criança, a família, a comunidade e o contexto em que vivem, dependendo assim, de um grande número de variáveis que devem ser observadas. Essas questões não foram objetivos desse estudo, mas podem ser consideradas em estudos futuros.

\section{Considerações finais}

Os resultados obtidos no presente estudo apontaram para serviços ainda centrados na criança. Diferentemente do que objetiva a IP, o que se pode observar é que os pais estão incluídos no tratamento dos filhos como informantes do histórico médico durante as triagens e entrevistas e no momento de receberem orientações das equipes multiprofissionais sobre como lidar com as crianças. Embora isso também seja importante, não contempla os objetivos da IP, uma vez que não está inserida no contexto da família e não preconiza a sua participação como ponto fundamental da intervenção. Há uma equipe interdisciplinar para os atendimentos necessários, todavia, sem formação específica na área, que implica em conhecimento intenso sobre desenvolvimento infantil, desde a avaliação até procedimentos de intervenção que sejam aplicáveis em todos os contextos de convivência da criança, reavaliando-a continuamente.

Observou-se, ainda, que a maioria das crianças usuárias dos serviços tem idade superior ao preconizado pelo critério de elegibilidade proposto pelas instituições ouvidas. Por outro lado, observou-se um número pequeno de bebês, indicando que chegam tarde ao serviço ou permanecem por longo tempo. Contudo, destaca-se a possibilidade das instituições em atender para além da faixa etária sugerida pelos documentos legais brasileiros, que é de três anos de idade.

Com relação às equipes interdisciplinares presentes em todas as APAES participantes, há divergência em princípios da IP, à qual postula a necessidade de formação específica. Esse indicador poderá ser um indicativo para políticas públicas na área de formação profissional, fundamentada na prioridade de atendimento especializado junto à primeira infância. Os respondentes evidenciaram o fato de muitos trabalharem com IP/EP há muitos anos, porém sem formação em nível de pós-graduação para este campo de atuação. Os dados apontaram para a necessidade da inserção da IP como disciplina obrigatória em todos os cursos de formação, seja ela inicial ou continuada. Isso viabilizaria 
garantir conhecimentos mínimos sobre o tema que, com a posterior complementação, aumentaria a efetividade dos serviços prestados a essas famílias.

Uma das limitações do estudo foi o pequeno número de instituições participantes, o que dificultou a generalização dos resultados obtidos. Em estudos futuros outras formas de coleta de dados poderiam ser utilizadas, como entrevista presencial, por exemplo. Estudos com populações maiores poderiam confirmar os dados obtidos neste trabalho. Uma outra limitação se refere ao tempo oferecido para responder o questionário (15 dias). Provavelmente, um tempo maior, com algum dispositivo de cobrança, poderia aumentar o número de respondentes. Sugere-se que pesquisas futuras possam incluir a informação sobre os tipos de serviços e apoio às famílias nos serviços de IP das APAES do estado de São Paulo, bem como uma questão de autoavaliação.

A IP é uma ferramenta importante para determinar estímulos e treinamentos adequados nos primeiros anos de vida, de forma a garantir à criança melhores condições de desenvolvimento. Definindo como será e qual capacidade desenvolverá a criança no decorrer de sua existência, esse processo favorecerá a interação mãe-bebê, vendo a IP como um subsídio para o desenvolvimento das potencialidades e dos sentidos remanescentes. Espera-se que os resultados obtidos com esse estudo impulsionem outros pesquisadores a dar continuidade em estudos nessa área, relevante para 0 desenvolvimento integral das crianças e qualidade de vida de suas famílias.

\section{Referências}

ALMEIDA, Isabel Chaves de et al. Práticas de intervenção precoce baseadas nas rotinas: Um projecto de formação e investigação. Análise Psicológica, Lisboa, v. 29, n. 1, p. 8398, 2011.

BAIRRÃO, Joaquim; ALMEIDA, Isabel Chaves de. Questões atuais em intervenção precoce. Psicologia, Lisboa, v. 17, n. 1, p. 15-29, 2003.

BOBREK, Andreia; GIL, Maria Stella Coutinho de Alcantara. Atuação e formação em estimulação precoce: Caracterização da percepção dos profissionais em três instituições. Revista Eletrônica de Educação, v. 10, n. 3, p. 121-137, 2016.

BOLSANELLO, Maria Augusta. Concepções sobre os procedimentos de intervenção e avaliação de profissionais em estimulação precoce. Educar em Revista, Curitiba, n. 22, p. 301-313, 2003. 
BORTAGARAI, Francine Manara et al. A interconsulta como dispositivo interdisciplinar em um grupo de intervenção precoce. Distúrbios de Comunicação, v. 27, n. 2, p. 392-400, 2015.

BRASIL. Ministério da Saúde. Conselho Nacional de Saúde. Resolução no 466, de 12 de dezembro de 2012. Brasília: Ministério da Saúde, 2012. Disponível em:

http://bvsms.saude.gov.br/bvs/saudelegis/cns/2013/res0466_12_12_2012.html. Acesso em: 13 ago. 2019.

BRASIL. Ministério da Saúde. Secretaria de Atenção à Saúde. Diretrizes de estimulação precoce: crianças de zero a 3 anos com atraso no desenvolvimento neuropsicomotor decorrente de microcefalia. Brasília: Ministério da Saúde, 2016. Disponível em:

http://bvsms.saude.gov.br/bvs/publicacoes/diretrizes_estimulacao_criancas_0a3anos_neu ropsicomotor.pdf. Acesso em: 05 ago. 2019.

CARNIEL, Camila Zorzetto et al. Influência de fatores de risco sobre o desenvolvimento da linguagem e contribuições da estimulação precoce: revisão integrativa da

literatura. Revista CEFAC, São Paulo, v. 19, n. 1, p. 109-118, 2017.

CORREA, Wesley; MINETTO, Maria de Fatima; CREPALDI, Maria Aparecida. Família como promotora do desenvolvimento de crianças que apresentam atrasos. Pensando famílias, Porto Alegre , v. 22, n. 1, p. 44-58, 2018.

DALMAU, Mariona et al. How to implement the family-centered model in early intervention. Análise Psicológica, Murcia, v. 33, n. 3, p. 641-651, 2017.

DUNST, Carl J. Factores de Risco e Oportunidade com Influência no Comportamento e Desenvolvimento da Criança e do Agregado Familiar. In: IV Encontro sobre Intervenção Precoce. Coimbra, 1995.

FEAPAES-SP - Federação das APAES do estado de São Paulo. A federação. Disponível em: http://feapaesp.org.br/federacao.asp?id=1. Acesso em: 07 ago. 2019.

FERREIRA, Tahena Silva et al. Intervenção precoce e a participação da família: relato de profissionais de APAES. Revista Educação Especial, Santa Maria, v. 32, p. 1-20, 2019.

FRANCO, Vitor. Dimensões transdisciplinares do trabalho de equipe em intervenção precoce. Interação em Psicologia, Curitiba, v. 11, n. 1, p. 113-121, 2007.

GARCIA, M. Pilar; MADRID, Dolores; GALANTE, Rafael. Children and Augmentative or Alternative Communication System (AACs): a perceptive vision of the role played by families and professionals. Anales de Psicologia, Murcia, v. 33, n. 2, p. 334-341, 2017.

GARCIA, Patrícia Azevedo et al. Influência de fatores de risco no desenvolvimento neuromotor de lactentes pré-termo no primeiro ano de vida. Revista Movimenta, Goiânia, v. 4, n. 2, p. 83-98, 2011.

GARCIA, Rosalba Maria Cardoso. Políticas para a educação especial e as formas organizativas do trabalho pedagógico. Revista Brasileira de Educação Especial, v. 12, n. 3, p. 299-316, 2006. 
GOLLO, Cristina; GRAVE, Magali Teresinha Quevedo. Incidência de crianças participantes dos programas de estimulação precoce de cinco Associações de Pais e Amigos dos Excepcionais do vale do Taquari. Caderno pedagógico, Lajeado, v. 12, n. 1, p. 221-230, 2015.

HALPERN, Ricardo et al. Fatores de risco para suspeita de atraso no desenvolvimento neuropsicomotor aos 12 meses de vida. Jornal de Pediatria, v. 76, n. 6, p. 421-428, 2000.

HANSEL, Ana Flávia; BOLSANELLO, Maria Augusta. As abordagens explicativas do desenvolvimento aplicadas à estimulação precoce. In: IX ANPED SUL, Seminário de Pesquisa em Educação da Região Sul, p. 1-9, 2012. Disponível em:

http://www.ucs.br/etc/conferencias/index.php/anpedsul/9anpedsul/paper/viewFile/2632/67 5. Acesso em: 13 ago. 2019.

IZIDORO, Isabela Rocha et al. Serviços especializados em intervenção precoce: elegibilidade e atuação multiprofissional. Revista CEFAC, São Paulo, v. 21, n. 4, e4919, 2019.

MARTINS, Paula Cristina. Protecção de Crianças e Jovens em Itinerários de Risco: representações sociais, modos e espaços. 2004. 492 f. Tese (Doutorado em Estudos da Criança) - Instituto de Estudos da Criança, Universidade do Minho, 2004. Disponível em: http://hdl.handle.net/1822/3238. Acesso em: 05 ago. 2019.

MENDES, Maria Elisabete da Silva Tomé. Avaliação da qualidade em intervenção precoce: Práticas no Distrito de Portalegre. 2010. 314 f. Tese (Doutorado em Psicologia) - Universidade do Porto, Faculdade de Psicologia e de Ciências da Educação, Porto, 2010. Disponível em: http://hdl.handle.net/10400.26/1549. Acesso em: 05 ago. 2019.

NASCIMENTO, Gabriela Cordeiro Corrêa do et al. Programas de intervenção precoce: caracterização de instituições e profissionais. Revista Educação Especial, Santa Maria, p. 431-446, 2018.

ORTIZ, Marilia Álvares; RIBEIRO, Maysa Ferreira Martins. Intervenção precoce e Síndrome de Down na APAE de Goiânia. Estudos, v. 40, n. 4, p. 461-478, 2013.

PACHECO, Raquel Cabral. Intervenção Precoce na Infância: Uma Abordagem ao Risco Ambiental na Região Autónoma dos Açores. 2013. 121 f. Dissertação (Mestrado em Ciências da Educação: Necessidades Educativas Especiais - Domínio Intervenção Precoce na Infância) - Universidade Fernando Pessoa, Porto, 2013. Disponível em: https://bdigital.ufp.pt/bitstream/10284/4061/1/Disserta\%C3\%A7\%C3\%A3o\%20Raquel\%20 Pacheco.pdf. Acesso em: 05 ago. 2019.

PADOVANI, Ricardo da Costa et al . Vulnerabilidade e bem-estar psicológicos do estudante universitário. Revista brasileira de terapias cognitivas, Rio de Janeiro, v. 10, n. 1, p. 02-10, 2014. 
PEREZ-LOPES, Julio. De La estimulacion precoz a La atención temprana: nuevas alternativas de actuación profesional. In: BOLSANELLO, Maria Augusta (Org.) Atenção e estimulação precoce: bebês com deficiência. Anais do I Simpósio Nacional de atenção e estimulação precoce. Curitiba, 2008, pp. 9-20.

PIMENTEL, Júlia Serpa. Avaliação de programas de intervenção precoce. Análise Psicológica, v. 1, n. 22, p. 43-54, 2004.

RAFANTE, Heulalia Charalo; SILVA, João Henrique; CAIADO, Katia Regina Moreno. A Federação Nacional das APAES no contexto da ditadura civil-militar no Brasil: Construção da hegemonia no campo da educação especial. Arquivos Analíticos de Políticas Educativas, v. 27, n. 64, 2019.

RIBEIRO, Débora Gerardo; PEROSA. Gimol Benzaquen; PADOVANI, Flávia Helena Pereira. Mental health, mother-child interaction and development at the end of the first year of life. Paidéia (Ribeirão Preto), v. 24, p. 331-339, 2017.

RODRIGUES, Olga Maria Piazentin Rolim; BOLSONI-SILVA, Alessandra Turini. Efeitos da prematuridade sobre o desenvolvimento de lactentes. Revista Brasileira de

Crescimento e Desenvolvimento Humano, v. 21, n. 1, p. 111-121, 2011.

SAMEROFF, Arnold; FIESE, Barbara F. Transactional regulation and early intervention. In: MEISELS, Samuel J.; SHONKOFF, Jack P. (Eds.). Handbook of Early Childhood Intervention. New York: Cambridge University Press, 1990, pp. 135-159.

SCHMIDT, Carlo et al. Intervenção precoce e autismo: um relato sobre o Programa SonRise. Psicologia revista, Belo Horizonte, v. 21, n. 2, p. 414-430, 2015.

SIMEONSSON, Rune J.; PEREIRA, Susana; SCARBOROUGH, Anita A. Documenting delay and disability in early development with the who - ICF. Psicologia, v. XVII, n. 1, p. 31-41, 2003.

SOARES, Diogo Miguel Cordeiro et al. Intervenção Precoce: intervenção junto da criança e da família. Trabalho de Conclusão de Curso. Universidade de Évora, 2012. Disponível em: http://www.psicologia.pt/artigos/textos/TL0290.pdf. Acesso em: 13 ago. 2019.

TEGETHOF, Maria Isabel Silva Chaves de Almeida. Estudos sobre a intervenção precoce em Portugal: ideias dos especialistas, dos profissionais e das famílias. 2007. 860 f. Tese (Doutorado em Psicologia) - Universidade do Porto, Faculdade de Psicologia e de Ciências da Educação, Porto, 2007. Disponível em:

http://hdl.handle.net/10400.12/47. Acesso em: 13 ago. 2019.

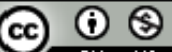

International (CC BY-NC 4.0) 